\title{
Adolescents' expression and perception of emotion in music reflects their broader abilities of emotional communication
}

Suvi Saarikallio*, Jonna Vuoskoski and Geoff Luck

\author{
* Correspondence: suvi.saarikallio@jyu.fi \\ Department of Music, University of \\ Jyväskylä, P.O. Box 35, Jyväskylä \\ 40014, Finland
}

\begin{abstract}
Background: Musical behavior has been shown to reflect broader individual differences. However, despite the prevalence of music in the lives of young people little is known about the mechanisms through which adolescents' musical behavior connects to their general socio-emotional behavior and adjustment. The current study focused on abilities of emotional communication and investigated whether adolescents' abilities in both perceiving and expressing emotions through music would be reflective of their general abilities of socio-emotional communication and interaction, measured through empathy and conduct problems. Due to the lack of previous research the study was mainly exploratory, but we expected accurate and congruent perception and expression of musical emotions to correlate positively with higher empathy and negatively with conduct problems.
\end{abstract}

Method: Sixty-one 14-year-olds (45 female, mean age 14.72) were given three music-related tasks that assessed emotion perception and emotion expression through music. Participants also filled in self-report scales for empathy (perspective taking and empathic concern) and conduct problems (externalized symptoms).

Results: The results showed that perspective taking was particularly related to accurate recognition of tenderness in music and congruent use of staccato articulation for the expression of anger through music. Empathic concern was particularly related to congruent use of slow tempo for expressing sadness and loud volume for expressing anger and also correlated with an overall tendency for intensified perception of fear in music. Externalized symptoms were particularly related to incongruent expression of sadness and anger through music: the use of staccato for expressing sadness and dull timbre for expressing anger.

Conclusion: Overall, the results preliminarily support the idea of using musical behavior as an indicator of the broader socio-emotional communication abilities, which in turn play a major role in adolescent adjustment and wellbeing.

Keywords: Adolescence; Music; Emotion perception; Emotion expression; Empathy; Conduct problems; Socio-emotional communication; Emotional intelligence

\section{Introduction}

\section{The relevance of emotions and empathy to wellbeing}

Emotions are a core aspect of human behavior and wellbeing (Gross, 1998; Mayer and Salovey 1997). They serve as the guiding structure of human behavior, directing our actions towards desired goals and away from adverse outcomes (Fridja 1988; Damasio

\section{(6) Springer}

(c) 2014 Saarikallio et al.; licensee Springer. This is an Open Access article distributed under the terms of the Creative Commons Attribution License (http://creativecommons.org/licenses/by/2.0), which permits unrestricted use, distribution, and reproduction in any medium, provided the original work is properly cited. 
1995). Emotion-related abilities and competencies - emotional intelligence - have been acknowledged as a major part of healthy functioning (Gross 1998; Mayer and Salovey 1997; Saarni 1999). The basic ability for emotion perception and recognition has been defined by concepts of emotion labeling (Swinkels and Giuliano 1995) or emotional clarity (Salovey et al. 1995), and regarding the relevance of this ability to health and wellbeing a differentiation is made between the mere tendency to monitor emotions, which is related to negative outcomes, and the ability to identify and label emotions, which is related to positive outcomes (Swinkels and Giuliano 1995). The ability for emotion recognition has also been proposed to serve as a fundamental for the more complex affect-related competencies that constitute emotional intelligence such as emotion regulation and the adaptive use of emotion in guiding social interaction (Gross 1998; Mayer and Salovey 1997). Indeed, emotion recognition in self and in others is shown to relate to empathy and prosocial behavior, adaptive coping strategies (Gohm 2003), experience of positive emotions, high self-esteem, greater satisfaction with social support (Swinkels and Giuliano 1995), as well as with lowered depression and social anxiety, fewer self-reports of somatic symptoms, and reduced cortisol secretion during induced stress episodes (Salovey et al. 2002). The findings indicate that although greater ability to distinguish emotions may actually result in increased experience of negative mood during a stress episode, this does not translate into an increased physiological arousal related to negative health outcomes (Salovey et al. 2002).

Recent research has begun to investigate empathy and the related mirror neuron theory as a relevant mechanism connecting the emotion recognition skills to adaptive social behavior (Gallese 2001; Iacoboni 2009). Empathy refers to responsiveness to the observed experiences of another person and consists of both affective and cognitive components (Davis 1980). It requires complex cognitive abilities related to perspective taking and understanding the feelings of others (Moore 1990) and develops relatively late across the years of adolescence (Eisenberg 1990; Eisenberg, et al., 1987; 1991; 1995). Both perspective taking and emotional concern increase significantly during adolescent development (Davis and Franzoi 1991), gradually leading to adult levels of being able to consider multiple perspectives and feel concern by late adolescence (Eisenberg 1990). Adolescents' level of empathy has been shown to relate to prosocial behavior such as altruism and helping (Eisenberg and Miller 1987; Eisenberg, et al., 1983; 1987; 1991; Eisenberg and Fabes 1999; Eisenberg and Fabes 1990), assertive behavior, and consideration for others (Garaigordobil 2009). Empathy is also thought to inhibit aggression through perspective taking and empathic concern (De Wied et al. 2010) and can be shown to have negative association with antisocial behavior, including aggression and delinquency (Jolliffe and Farrington 2004; Miller and Eisenberg 1988; Feschbach and Feschbach 2009; Garaigordobil 2009). Indeed, the lack of empathy has been considered to be a risk factor for disruptive behavior disorders (De Wied et al. 2010). Emotionrelated skills such as emotion recognition and empathy can thus be considered major factors in adolescent development regarding social communication and wellbeing.

\section{Music as emotional communication}

Music serves as a useful forum for studying emotion: musical communication is somewhat isomorphic to general communication of emotion: music involves interaction through abstract patterns that are emotionally meaningful to other humans (Dissanayake 2008). 
The communication of emotion through music employs the same mechanisms than communication of emotion in general: for instance, emotional contagion, a subcomponent of empathy, has been proposed to be one of the core mechanisms through which music induces emotions in listeners (Juslin and Västfjäll 2008). This gives rise to the idea of testing whether abilities of communicating (both perceiving and expressing) emotions through music would relate to the general abilities of emotional communication and interaction. It has been proposed that emotional engagement in music may increase the 'awareness of feelings', i.e. the ability to experience various emotional nuances, express various degrees of intensity of emotions, and maintain precise concepts about feelings and that this ability in music might transfer to general emotional life (Ruud 1997). However, little empirical work on the topic exists.

Music has been shown to accurately communicate basic emotions and previous studies have identified particular musical features (e.g., tempo, sound level, timbre) that relate to the expression of discrete emotions such as happiness, sadness, anger, fear, and tenderness in music (Gabrielsson and Lindström 2001). Extensive reviews (Juslin and Timmers 2010; Juslin and Laukka, 2003; 2004; Gabrielsson and Lindström 2010) show that happiness is typically characterized by fast tempo, major mode, medium-high or high sound level, high pitch, staccato articulation, and bright timbre, that sadness is characterized by slow tempo, minor mode, low sound level, low pitch, legato articulation, and dull timbre, and that anger is characterized by fast tempo, high sound level, high pitch, staccato articulation, and sharp timbre and spectral noise. These features share a high similarity with the vocal expression of emotion (Juslin and Laukka 2003), and, overall, the agreement between individuals regarding the connection between the abovementioned musical features and perceived emotions appears extremely high (Bigand et al. 2006; Fredrickson 2000; Juslin 1997; Juslin and Laukka 2004; Juslin and Timmers 2010; Vieillard et al. 2008).

\section{Musical emotions reflect individual differences}

Emotion recognition in music also appears to be influenced by a range of top-down processes that relate to various individual differences in personality and emotionality. Personality traits of extraversion, openness, and agreeableness have been shown to correlate with higher ratings of perceived positive, and lower ratings of perceived negative, emotions in music, while neuroticism relates to an opposite pattern (Liljeström 2011; Vuoskoski and Eerola 2011a). A similar bias in perception has also been observed for mood: state negative mood (Vuoskoski and Eerola 2011a) and clinical depression (Punkanen et al. 2011) correlate with higher ratings of perceived negative, and lower ratings of perceived positive, emotion in music. As regards emotion recognition accuracy, certain aspects of emotional intelligence (perceiving emotions and using emotions to facilitate thought) have been found to relate to better identification of musicians' intended expression of emotion (Resnicow et al. 2004). Similar findings have been observed for empathy: participants' scores on the affective and overall empathy (not on cognitive empathy) have been shown to correlate with better accuracy of recognizing the intended emotion in music (Wöllner 2012). Preliminary findings also indicate a correlation between empathy and more intense emotional responding to music, (Vuoskoski and Eerola 2011b, 2012). Overall, the observed tendencies in musical emotion perception appear state and trait-congruent, i.e. the musical behavior is in accordance with the observed affective state and/or the long-term 
behavioral trait of the individual. This gives further support to the idea of using musical behavior as an indicator of general affective behavior.

\section{Musical emotions in adolescence}

While there is emerging evidence that emotional communication through music indeed is reflective of broader socio-emotional behavior, little is known about these connections during adolescence. This is a serious limitation, as adolescence in particular is a period during which music plays a major role, and has been shown to serve as a medium for adolescents' emotional self-regulation, peer-group affiliation, and identity formation (Arnett 1995; Behne 1997; Laiho 2004; North, et al., 2000; Saarikallio and Erkkilä 2007). The particular ability to perceive emotion in music begins to develop early, with 5-7-month-olds already showing a preference for happy over sad expression (Nawrot 2003), three-year-olds distinguishing between happy and sad music (Kastner and Crowder 1990), and 4-6-year olds correctly identifying happiness, sadness, anger, and fear in music (Dolgin and Adelson 1990; Cunningham and Sterling 1988). Regarding emotion expression, children as young as four are able to manipulate tempo, dynamics, and pitch to create happy or sad expression (Adachi and Trehub 1998). From these, it can be concluded that the perception of happiness and sadness develops relatively early and precedes the perception of more complex emotions such as anger and fear. Furthermore, it has been proposed that children first learn to identify musical emotions using the more universal features of music (e.g. tempo, loudness) that contain similarity to vocal expression, while the use of culturally learned features in music (e.g. mode) develops later through social learning (Schubert and McPherson 2006). However, knowledge about the possible connections of these musical abilities to the general emotion-related competencies and tendencies like empathy in children and adolescents is virtually non-existent.

\section{Aims of the study}

While emerging evidence suggests that emotion perception in music reflects broader individual differences in behavior, there are several major gaps in the current literature. The existent studies on this topic have focused almost solely on emotion perception, neglecting the expression of emotion through music. Furthermore, most research on musical emotion perception and expression has been conducted with adults, despite the prevalence of music in the lives of young people. Therefore, this study aimed to investigate adolescents' individual differences in both perception and expression of emotion in music and to test whether these abilities of musical communication of emotion would be reflective of the adolescents' broader abilities of socio-emotional communication and interaction. Indeed, as individual differences in emotion recognition in general are known to relate to empathy and prosocial behavior (e.g. Gohm 2003), we expected also music-related abilities for emotion recognition and expression to be reflective of these. Skills in musical communication of emotion were assessed through 1) accuracy of emotion recognition in music, 2) general tendencies in musical emotion perception, and 3) congruent use of musical parameters in the expression of emotions. The general abilities of socio-emotional communication and interaction were measured through empathy (a feature of socio-emotional communication that further relates to 
psychological adjustment) and conduct problems (an feature that relates to psychological maladjustment). Due to the lack of previous research the study was primarily exploratory, but we did expect accurate and congruent perception and expression of musical emotions to correlate positively with empathy and negatively with conduct problems. In detail, we hypothesized that higher empathy would correlate with more accurate recognition of intended emotion in music (see Resnicow et al., 2004; Wöllner 2012), with an overall trait-congruent tendency of compassionate and intense perception of emotion in music (see Ladinig and Schellenberg 2012 Liljeström 2011; Vuoskoski and Eerola 2011a, 2012), and with a congruent use of musical parameters in the expression of emotion (e.g. expressing happiness with high tempo and sadness with slow tempo, see Juslin and Timmers 2010; Juslin and Laukka 2003; 2004; Gabrielsson \& Lindström, 2010). In addition, individuals with conduct problems were expected to demonstrate a pattern opposite to previous hypotheses: lower accuracy in emotion recognition, less compassionate and intense perception of emotion, and incongruent use of musical parameters in the expression. Finally, we expected to find effects particularly for the more complex emotions (anger, fear, tenderness) in comparison to happiness and sadness, which six-month-olds already recognize (Nawrot 2003).

\section{Method}

\section{Participants}

A total of 61 adolescents (45 females, 16 males) took part in the study. All participants were 8-graders with a mean age 14.72 (SD: .45). Recruitment was conducted in local schools and ethical clearances, permissions from school officials, and informed consents from both the students and their parents were obtained prior to the study. All participants were white, Finnish-speaking adolescents from public schools and due to small differences in the socioeconomic statuses within the Finnish population in general, further SES details were not obtained. As regards music background, approximately half of the participants $(n=32)$ had received private music lessons while half $(\mathrm{n}=29)$ had not. Motivation for participation was not directly asked, but based on free comments it appeared to vary from interest in the study to using the opportunity to have a day off school. The participants also received movie tickets as a reward for participation. Due to the lack of previous research and reliable estimates of expected effect sizes no power analysis regarding desired sample size was conducted. The sample was considered small, but sufficient for the exploratory nature of the study. The University of Jyväskylä Ethical Committee approved the study.

\section{Measures for empathy and conduct problems}

Age-appropriate and previously validated self-report scales were selected for assessing adolescents' level of empathy and conduct problems. Empathy was measured with the empathic concern and perspective taking subscales of the Interpersonal Reactivity Index, a self-report measure of dispositional empathy (IRI; Davis 1980; 1983). The Empathic Concern (EC) subscale was chosen, because it assesses sympathetic and compassionate responding towards others, and is shown to be the most relevant sub-component of empathy for prosocial behavior (Eisenberg and Fabes 1990). In addition, we used the Perspective Taking (PT) subscale measuring the more cognitive tendency to adopt other peoples' viewpoints, because we assumed this tendency to be relevant for the accurate 
(congruent with other's opinions) recognition of emotion in music. Furthermore, empathic concern and perspective taking are the two sub-components of empathy that have been shown to most increase over the course of adolescent years being particularly relevant for the development of adult levels of understanding multiple perspectives and feeling concern for others (Davis and Franzoi 1991). Both sub-scales consist of 7 items, such as "I sometimes try to understand my friends better by imagining how things look from their perspective" (PT). The items are answered on a 5-point scale ranging from does not describe me well to describes me very well. Previous research (Davis 1983) has reported internal consistency reliabilities around .70 for both scales, but in the current sample the Cronbach's alpha coefficients were relatively poor, only .59 for empathic concern, and .58 for perspective taking. However, as lower alpha coefficients are more acceptable in non-clinical, exploratory studies (Bland and Altman 1997), the scales were considered appropriate for this study.

Conduct problems were measured with the externalized symptoms subscale of the Strengths and Difficulties Questionnaire (SDQ; Goodman et al. 1998). SDQ is a selfreport measure for screening for behavioral and emotional problems with children and adolescents, originally consisting of 5 subscales. Two of the subscales, conduct problems and hyperactivity, assess problems in external social behavior and conduct, and can be combined into a unified scale of Externalized Symptoms (ES) (Goodman et al. 2010). Overall, the externalized symptoms subscale measures a tendency to break rules, fight, lose temper, and be restless and hasty, and was therefore considered appropriate for measuring disruptive socio-emotional behavior particularly in terms of emotional communication towards others. The scale consists of ten items such as "I get very angry and often lose my temper", which are answered on a 3-point scale with options Not True, Somewhat True, and Certainly True. The Cronbach's alpha coefficient for the externalized symptoms scale in the current sample was .72.

\section{Accurate emotion recognition in music}

The first musical task was designed to measure the ability to accurately recognize emotions expressed by music. For reliable measurement of accurate perception it was vital to choose stimuli that could be considered indisputably expressive of the intended emotion. The music excerpts were therefore carefully pre-selected to be expressive of the five basic emotions most used in music research, happiness, sadness, anger, fear, and tenderness (Juslin and Timmers 2010; Juslin and Laukka 2003; 2004). A panel of three music and emotion researchers with expert knowledge on the musical features that have been found representative of different emotions (Juslin and Timmers 2010; Juslin and Laukka 2003; 2004; Gabrielsson and Lindström 2010) first pre-selected a preliminary set of 60-second long excerpts of pop music. The panel then rated each excerpt regarding their appropriateness, and selected 3 excerpts most illustrative of each target emotion, resulting in 15 excerpts in total. In order to ensure inter-rater agreement only excerpts that all evaluators agreed upon were chosen: each chosen excerpt needed to receive an appropriateness rating of 4 or 5 on a scale of $1-5$ from each evaluator.

During the experiment participants were presented with the 15 music excerpts through loudspeakers in a different random order for every participant. Participants were asked to select which of the abovementioned basic emotions they found each musical excerpt to be most representative. Percentage accuracy with intended emotion was calculated based on 
each participant's emotion selection for each excerpt, and the accuracy score was used as a measure of emotion recognition accuracy in music for each emotion. Familiarity for each excerpt was also checked (on a five-point scale), but did not correlate with the perception accuracy for any of the emotions.

\section{Overall tendencies in musical emotion perception}

The second task was designed to measure general, possibly trait-congruent, tendencies in the emotions perceived in music. The stimuli for this task were selected from a set of music from movie soundtracks: the chosen 50 excerpts were short (15 s) and had previously been rated to be unfamiliar and expressive of basic emotions (happiness, sadness, anger, fear, tenderness) with a varying degree of clarity/conformity (excerpts 001-050 from Eerola and Vuoskoski 2011). For this task, it was not purposeful to only choose excerpts highly representative of certain emotions, but rather excerpts susceptible to a wider range of interpretation in order to provide more room for the occurrence of top-down biases in perception. Therefore, the set of the 50 chosen excerpts consisted of only 20 excerpts previously rated (Eerola and Vuoskoski 2011) as clearly illustrative of happiness, sadness, anger, fear, and tenderness and of 30 excerpts previously rated as only moderately expressive of these emotions.

The list of the emotion words used to rate the excerpts was extended beyond the most common five basic emotions, because previous research has stressed that individual musical experiences consist of a wide range of affective nuances (Juslin and Laukka 2004; Zentner and Scherer 2008). The initial list of emotion words was formulated based on previous research with adults (Juslin and Laukka 2004; Zentner and Scherer 2008) and qualitative descriptions about musical emotions collected from 20 adolescents (14-year-olds), The original list consisted of 11 emotion words: happiness/joy, sadness, anger, fear, tenderness, longing, power, hope, serenity, anxiety, and carefreeness. A pilot study was conducted to test them: 30 music students rated the 50 music excerpts on how much they were expressive of these 11 emotions (on a 9-point scale ranging from not at all to extremely lot). Three of the words showed stronger than .90 correlations to other words (serenity with tenderness, anxiety with fear, and carefreeness with happiness/joy) and were therefore removed as redundant. The final list of emotion words then consisted of the following eight emotions: happiness/joy, sadness, anger, fear, tenderness, longing, power, and hope.

During the experiment the participants listened to the music excerpts played through headphones (in a different random order for every participant). Each music excerpt was rated on the abovementioned emotion words on a 7-point scale ranging from not at all to very much. Mean emotion ratings were calculated for each emotion for each participant using ratings given for all 50 excerpts (i.e., the mean sadness rating of a participant was calculated from that participant's sadness ratings for all 50 excerpts). This procedure enabled the investigation of overall tendencies in the whole stimulus set, as has been used in previous studies (Isen and Shalker 1982; Bouhuys et al., 1995; Vuoskoski and Eerola 2011a).

\section{Emotion expression}

The third task was designed to investigate how adolescents would use musical parameters to express basic emotions. We measured the use of five parameters (tempo, loudness, 
pitch, articulation, and timbre) for the expression of three emotions (happiness, sadness, anger). The adolescents were presented with three excerpts, instrumental versions of children's song melodies, including no lyrics or accompaniment (in a different random order for each participant). The duration of the excerpts was 30s, after which playback was automatically repeated. The participants created three renditions for each excerpt: happy, sad and angry, thus resulting in nine renditions in total. The musical parameters were modified through an interface called the Music Box, which includes separate sliders for each parameter and enables modification of the musical material in real time (Bresin and Friberg 2011). The slider movements were further converted into numerical form with Pure Data software, and the scales for the parameters were: $0-5$ for tempo (slow-fast), $-10-10$ for loudness (quiet-loud), -24-24 for pitch (low-high), $0-5$ for articulation (legato-staccato), and $0-3$ for timbre (dull-bright). The participants could render each excerpt as long as they wanted, after which the numeric representations of the slider positions were saved as the measure of the participants' opinion for optimal expression.

\section{Statistical analyses}

Ratings from the music tasks were correlated with the measures of socio-emotional behavior. Stepwise regression analyses were conducted separately for perspective taking, empathic concern, and externalized symptoms in order to identify the most relevant music-related emotional competencies and tendencies in predicting each of them. The statistical analyses were conducted using PASW Statistics, 18.0.

\section{Results}

\section{Measures of musical behavior: recognition accuracy, perceptual tendencies, and emotional expression}

The accuracy of emotion recognition was generally high, with $76 \%$ of all music excerpts correctly identified. The mean emotion ratings for each intended emotion are illustrated in Figure 1. The accuracy was 98\% for happiness, $72 \%$ for sadness, $59 \%$ for anger, $73 \%$ for fear, and $77 \%$ for tenderness. The extremely high mean accuracy for happiness (98\%) meant that basically all adolescents correctly recognized the intended happy excerpts as being happy. While this was a positive result in terms of adolescents' emotion perception ability, it also meant lack of variance in happiness score, which was therefore excluded from further analyses. As regards inaccuracy patterns, the sad excerpts not identified as sad were mainly interpreted as being tender, angry excerpts as being happy, fearful excerpt as being angry and tender excerpts as being either happy or sad (see Figure 1).

The mean ratings for each emotion in the second task (measuring perception tendencies) are listed in Table 1. Fear received the highest mean scores, indicating that the stimulus set was generally considered most reflective of fear. This overall bias may be due to the fact that 20 of the 50 excerpts were selected to be expressive of negative, high-arousal emotions (fear or anger).

Table 2 shows the mean scores for each musical parameter for expressing happiness, sadness and anger. Happiness was generally expressed through high tempo, high pitch, loud volume, staccato-type articulation, and bright timbre, sadness through slow tempo, quiet volume, relatively low pitch, legato articulation, and dull timbre, and anger through moderately high tempo, loud volume, low pitch, legato articulation, and dull timbre. 


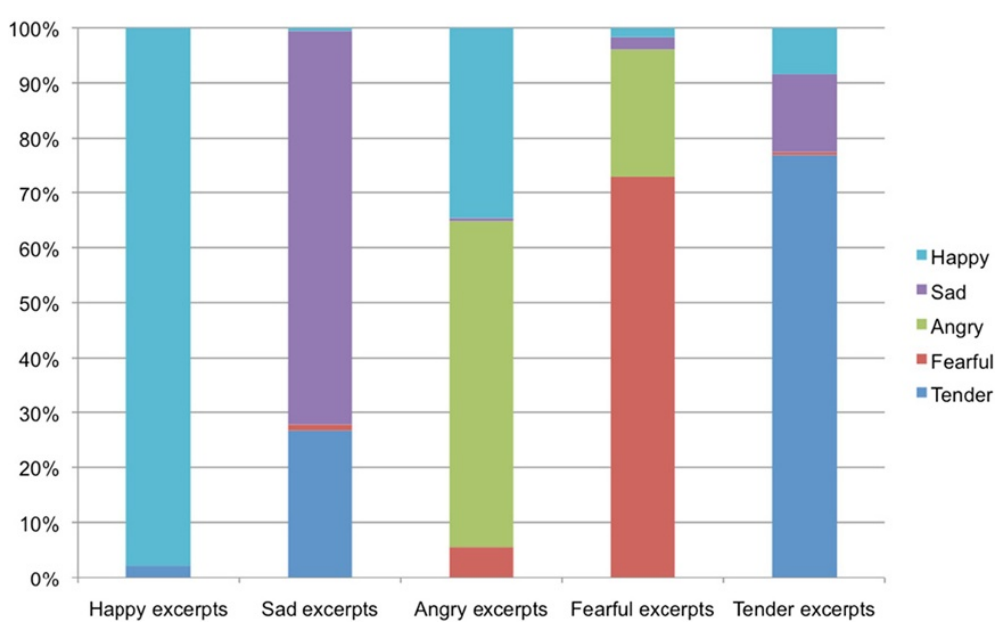

Figure 1 Percentages of the identified emotions for the happy, sad, angry, fearful, and tender excerpts.

\section{Correlations between the measures}

Measures of socio-emotional behavior correlated as expected with each other. Empathic concern and perspective taking correlated significantly $(r=.33 ; p \leq .01)$. A significant negative correlation was found between perspective taking and externalized symptoms $(r=-.34 ; p \leq .01)$, while the negative correlation between empathic concern and externalized symptoms remained non-significant $(r=-.19 ; p=.15)$. No differences were found for any of the measures based on gender, music lessons, or age.

The correlations between the measures for musical behavior and the measures of socio-emotional behavior are shown in Table 3. The effect-sizes of the correlations were generally moderate (Cohen 1988). Perspective taking correlated relatively strongly with more accurate recognition of tenderness in music $(r=.40 ; p \leq .01)$ and demonstrated medium-sized correlations to expressing anger with louder volume $(r=.28 ; p<.05)$ and higher pitch $(r=.31 ; p<.05)$. Empathic concern was not correlated with recognition accuracy but showed a moderate correlation to a general tendency of giving higher ratings for fear $(r=.27 ; p \leq .05)$ and hope $(r=.28 ; p \leq .05)$ in music. Furthermore, empathic concern correlated moderately with the use of slower tempi for the expression of sadness $(r=-.30 ; p<.05)$ and the use of louder volume for the expression of happiness $(r=.29 ; p<.05)$ and anger $(r=.31 ; p<.05)$. Externalized symptoms also did not correlate with recognition accuracy but were moderately negatively correlated with

Table 1 Mean ratings for perceived emotions in music

\begin{tabular}{lll}
\hline Emotion & Mean & S.D \\
\hline Happiness & 4.37 & 2.30 \\
Tenderness & 4.32 & 2.22 \\
Anger & 3.56 & 1.98 \\
Sadness & 3.73 & 2.36 \\
Fear & 5.07 & 2.15 \\
Longing & 4.66 & 2.51 \\
Hope & 4.34 & 2.20 \\
Power & 4.37 & 2.21 \\
\hline
\end{tabular}


Table 2 Mean scores for the musical parameters in expressing happiness, sadness and anger

\begin{tabular}{|c|c|c|c|c|c|c|}
\hline & \multicolumn{2}{|c|}{ Happiness } & \multicolumn{2}{|c|}{ Sadness } & \multicolumn{2}{|l|}{ Anger } \\
\hline & $M$ & $S D$ & $M$ & $S D$ & $M$ & $S D$ \\
\hline Tempo & 2.11 & 3.26 & 0.80 & 0.33 & 1.52 & 0.75 \\
\hline Loudness & 3.31 & 7.93 & -3.17 & 3.24 & 2.00 & 5.34 \\
\hline Pitch & 6.19 & 1.00 & -11.99 & 5.98 & -17.90 & 6.63 \\
\hline Articulation & 2.60 & 0.55 & 1.89 & 1.03 & 1.67 & 1.64 \\
\hline Timbre & 1.72 & 0.55 & 0.90 & 0.50 & 0.91 & 0.71 \\
\hline
\end{tabular}

the general perception of hope in music $(\mathrm{r}=-.27 ; \mathrm{p} \leq .05)$ and also moderately correlated with the expression of anger through more dull timbre $(r=-.31 ; p=.05)$.

\section{Musical behavior in predicting socio-emotional behavior}

The most relevant musical indicators for the measures of socio-emotional communication abilities were further investigated through stepwise regression analyses. Significant predictors for perspective taking, empathic concern, and externalized symptoms are listed in Table 4. Perspective taking was significantly predicted by the accurate identification of tenderness in music and by the expression of anger with staccato articulation. These two predictors explained a significant proportion of variance in the perspective taking scores, $R^{2}=.25$, $F(2,58)=8.76, p<.001$. Empathic concern was significantly predicted by the expression of anger through loud volume, the expression of sadness with slow tempo, and a general tendency of perceiving greater amount of fear in music. These three predictors explained a significant proportion of variance in the empathic concern scores, $R^{2}=.27, F(3,57)=6.44$, $p<.001$. As regards externalized symptoms, two music-related measures, the expression of anger with more dull timbre and the expression of sadness through staccato articulation, appeared as significant predictors that explained a significant proportion of variance in the externalized symptoms score, $R^{2}=.19, F(2,58)=6.11, p<.01$.

\section{Discussion}

This study explored whether adolescents' abilities and tendencies in musical communication of emotion were reflective of their broader socio-emotional communication and interaction. The results showed that particular features of music-related emotional communication were indeed reflective of both empathy and conduct problems. In particular, perspective taking, the cognitive component of empathy, was related to the accurate recognition of tenderness in music and the expression of anger with staccato articulation. Empathic concern, the affective component of empathy, was particularly related to a general tendency for perceiving more fear in the music and expressing anger with loud volume and sadness with slow tempo. Externalized symptoms were particularly related to the expression of anger with dull timbre and sadness with staccato articulation. Overall, the findings were consistent with hypothesized directions, as they connected empathy with more congruent recognition and expression of emotion in music and conduct problems to opposing patterns. However, these connections were not consistently found for all musical features and all emotions. Overall, the results thus provide preliminary support for the proposal that musical behavior can be used as an indicator of broader socio-emotional communication abilities. The findings also indicate that instead of a general pattern of congruence versus Incongruence, a more 
Table 3 Correlations (Pearson) between the measures of musical behavior - a) recognition accuracy for sadness, anger, fear, and tenderness in music, b) overall tendency of perceiving different emotions in music, and c) use of different musical parameters for expressing happiness, sadness, and anger - and the measures of socio-emotional behavior: perspective taking (PT), empathic concern (EC), and externalized symptoms (ES)

\begin{tabular}{|c|c|c|c|}
\hline Musical behavior measures & PT & $\mathrm{EC}$ & ES \\
\hline \multicolumn{4}{|l|}{ a) Emotion recognition accuracy: } \\
\hline Sadness & -.01 & -.04 & -.00 \\
\hline Anger & -.11 & .24 & -.01 \\
\hline Fear & .01 & -.00 & -.03 \\
\hline Tenderness & $.40^{* *}$ & .05 & -.15 \\
\hline \multicolumn{4}{|c|}{ b) Emotion perception tendencies: } \\
\hline Happiness & .02 & -.10 & -.04 \\
\hline Tenderness & .13 & .08 & -.24 \\
\hline Anger & .02 & .02 & .04 \\
\hline Sadness & .18 & .03 & -.09 \\
\hline Fear & .23 & $.27^{*}$ & -.16 \\
\hline Longing & .10 & -.02 & -.05 \\
\hline Hope & .10 & $.28^{*}$ & $-.27^{*}$ \\
\hline Power & .08 & .07 & -.17 \\
\hline \multicolumn{4}{|l|}{ c) Emotional expression: } \\
\hline Tempo happy & .03 & .06 & -.05 \\
\hline Tempo sad & .13 & $-.30^{*}$ & -.12 \\
\hline Tempo angry & -.08 & .05 & .09 \\
\hline Loudness happy & -.00 & $.29^{*}$ & .02 \\
\hline Loudness sad & .05 & -.02 & .05 \\
\hline Loudness angry & $.28^{*}$ & $.31^{*}$ & -.18 \\
\hline Pitch happy & -.14 & .14 & .06 \\
\hline Pitch sad & -.02 & -.05 & -.02 \\
\hline Pitch angry & $.30^{*}$ & .02 & -.14 \\
\hline Articulation happy & -.07 & .21 & -.01 \\
\hline Articulation sad & .03 & .15 & .20 \\
\hline Articulation angry & .19 & .11 & -.10 \\
\hline Timbre happy & .05 & .02 & -.07 \\
\hline Timbre sad & .00 & -.01 & .03 \\
\hline Timbre angry & .24 & -.05 & $-.31^{*}$ \\
\hline
\end{tabular}

${ }^{* *} \mathrm{p}<.01,{ }^{*} \mathrm{p}<.05$.

detailed approach is needed to fully understand the links between emotional abilities in music and in general.

As regards to the perceptual versus expressive features of musical communication, features related to the expression of emotion through music appeared as significant predictors for all measures of socio-emotional communication. This is not surprising, as the study focused on emotional communication, of which expression is a major part, and it points to the relevance of investigating musical behavior in a comprehensive manner, not only focusing on the perception but also on the expressive side of communication. The findings indicated that adolescents employ musical parameters generally in line with 
Table 4 Standardized beta coefficients for the significant predictors (measures of musical behavior) for perspective taking, empathic concern, and externalized symptoms

\begin{tabular}{llll}
\hline & $\boldsymbol{\beta}$ & $\mathbf{t}$ & $\mathbf{p}$ \\
\hline Perspective taking & & & \\
$\quad$ Perception accuracy, tenderness & .47 & 3.86 & .000 \\
$\quad$ Expression, articulation for anger & .30 & 2.48 & .017 \\
Empathic concern & & & \\
$\quad$ Expression, loudness for anger & .34 & 2.88 & .006 \\
Expression, tempo for sadness & -.31 & -2.64 & .011 \\
Perception tendency, fear & .24 & 2.02 & .048 \\
Externalized symptoms & & & .005 \\
$\quad$ Expression, timbre for anger & -.36 & -2.91 & .024 \\
$\quad$ Expression, articulation for sadness & .29 & 2.33 & \\
\hline
\end{tabular}

what has been demonstrated in previous studies with adults (Bresin and Friberg 2011; Juslin and Timmers 2010; Juslin and Laukka 2003; 2004). Furthermore, the congruent use of these musical parameters (staccato articulation and loud volume for anger, slow tempo for sadness) predicted empathy while incongruent use of the parameters (dull timbre for anger and staccato articulation for sadness) predicted externalized symptoms.

With regards to the different emotions in musical communication, effects were observed for anger, fear, sadness, and tenderness. This is somewhat in line with previous research showing that the ability to perceive and express complex emotions such as anger and fear in music develops later than the ability of distinguishing between happy and sad expression (Dolgin and Adelson 1990; Cunningham and Sterling 1988; Kastner and Crowder 1990; Schubert and McPherson 2006). It seems logical that differences in socio-emotional abilities would manifest themselves particularly in the more refined nuances of musical expression. Furthermore, it may be that the emotions found central to this study particularly reflect the socio-emotional concepts chosen for investigation. The relevance of tenderness may be due to trait-congruence with empathy. For instance, agreeable people are often high in empathy and define themselves as typically tender-minded, sympathetic, altruistic, and trustful (e.g.,John and Srivastava 1999). Meanwhile, the symptomatology around conduct problems typically relates to aggression and delinquency, which can be considered opposites for tender and empathic behavior (De Wied et al., 2010; Jolliffe and Farrington 2004; Miller and Eisenberg, 1988; Feschbach and Feschbach 2009; Garaigordobil 2009), and this may explain the role of sadness, anger, and fear as relevant emotions among the significant musical predictor variables.

Different musical predictors were also found to be significant for the two subcomponents of empathy. Perspective taking was significantly predicted by the perception accuracy for tenderness, while empathic concern was significantly predicted by an overall tendency of perceiving more fear in music. This suggests that, in the emotion perception of music, cognitive empathy may be particularly reflected through the accuracy of musical perception, while affective empathy is rather reflected through trait-congruent perceptual biases or tendencies. This observation is well in line with how the two sub-components are defined: perspective taking referring to the ability for adopting perspectives of others and empathic concern referring to the ability to feel concern for others (Davis 1980; 1983). 
The effect sizes were not notably strong - the effects were generally low to moderate. However, this is common in studies investigating connections between general emotionality and emotional responses to music (Ladinig and Schellenberg 2012; Liljeström 2011; Vuoskoski and Eerola 2011a). Furthermore, we did not use a sample particularly screened for particular individual features (such as high empathy, high conduct problems) and stronger effects could be expected if particular, even clinical, groups were identified and compared to non-clinical individuals. All participants were $8^{\text {th }}$ graders (14-15-year-olds) and thus representative of a relatively short timewindow of adolescent development. Abilities for empathy and prosocial behavior slowly develop over the course of adolescence (Davis and Franzoi 1991; Eisenberg 1990), during the very years when music is known to play a major role in identity development, emotion regulation, and social group integration (Arnett 1995; Behne 1997; Laiho 2004; North et al., 2000; Saarikallio and Erkkilä 2007). Connections between the musical and the general skills of emotional communication may thus significantly vary at different developmental phases and between the more musically engaged and non-engaged adolescents. Differences based on gender or music background were not observed in the current study, but we did not particularly select highly musically engaged versus non-engaged participants. In addition, the sample consisted of more females than males, not allowing a reliable gender comparison and generally being more illustrative of adolescent girls than boys. Therefore, while current results demonstrate the existence of connections between adolescents' musical communication of emotion and general socio-emotional communication, they are very limited in scope in terms of interactions related to developmental change and individual differences.

Empathy and conduct problems are negatively correlated and can be considered opposing in terms of their connections to adolescent psychological adjustment and wellbeing (De Wied et al., 2010; Jolliffe and Farrington 2004; Miller \& Eisenberg 1988; Feschbach and Feschbach 2009; Garaigordobil 2009). The current findings are therefore relevant to our understanding of wellbeing in adolescence. The results demonstrate abilities of emotional communication as a link that connects adolescents' musical behavior to broader socio-emotional behavior. However, the results are also limited in their scope: they particularly focus on the communicative aspects of emotional competence, both in musical behavior (perception and expression of emotion through music) and in socio-emotional behavior (empathic versus abrupt and confrontational socio-emotional interaction). Thus, other emotion-related competencies such as the self-regulation of emotion and its possible relevance for internalized symptomatology are excluded from the scope of this work. Music is known to be an important means for mood regulation for adolescents (Saarikallio and Erkkilä 2007) and emotion regulation strategies and coping styles have been proposed to play a role in understanding the health-impact of music (Miranda et al. 2012). Therefore, even broader perspectives are required for comprehensive understanding of how different emotional competencies serve as a link between musical and general behavior. Nonetheless, the current findings are an important preliminary step in clarifying how the particular features of emotional communication connect adolescents' musical engagement to their broader socio-emotional competence and further to their wellbeing. 


\section{Competing interests}

The authors declare that they have no competing interests.

\section{Authors' contributions}

SS designed the study, participated in data collection and analysis of all tasks and wrote the manuscript. JV carried out the listening test measuring tendencies of musical emotion perception and participated in related analyses. GL carried out the listening test measuring emotion recognition in music and participated in related analyses. All authors read and approved the final manuscript.

Received: 23 April 2014 Accepted: 15 September 2014

Published online: 18 December 2014

\section{References}

Adachi, M, \& Trehub, SE. (1998). Children's expression of emotion in song. Psychology of Music, 26(2), 133-153. Arnett, JJ. (1995). Adolescents' uses of media for self-socialization. Journal of Youth and Adolescence, 24(5), 535-549. Behne, K. (1997). The Development of "Musicerleben"in Adolescence: How and why Young People Listen to Music. In I Deliége \& JA Sloboda (Eds.), Perception and Cognition of Music. Hove, UK: Psychology Press.

Bigand, E, Vieillard, S, Madurell, F, Marozeau, J, \& Dacquet, A. (2006). Multidimensional scaling of emotional responses to music: the effect of musical expertise and of the duration of the excerpts. Cognition and Emotion, 19, 1113-1139. Bland, JM, \& Altman, DG. (1997). Statistics notes: Cronbach's alpha. BMJ, 314, 572.

Bouhuys, AL, Bloem, GM, \& Groothuis, TGG. (1995). Induction of depressed and elated mood by music influences the perception of facial emotional expressions in healthy subjects. Journal of Affective Disorders, 33(4), 215-226.

Bresin, R, \& Friberg, A. (2011). Emotion rendering in music: range and characteristic values of seven musical variables. Cortex, 47(9), 1068-1081.

Cohen, J. (1988). Statistical Power Analysis for the Behavioral Sciences (2nd ed.). Hillsdale: New Jersey: Lawrence Erlbaum Associates.

Cunningham, JG, \& Sterling, RS. (1988). Developmental change in the understanding of affective meaning in music. Motivation and Emotion, 12, 399-413.

Damasio, AR. (1995). Descartes' Error: Emotion, Reason, and the Human Brain. New York: Picador: Avon Books.

Davis, MH. (1980). A multidimensional approach to individual differences in empathy. JSAS Catalog of Selected Documents in Psychology, 10, 85.

Davis, MH. (1983). Measuring individual differences in empathy: evidence for a multidimensional approach. Journal of Personality and Social Psychology, 44, 113-126.

Davis, MH, \& Franzoi, SL. (1991). Stability and change in adolescent self-consciousness and empathy. Journal of Research in Personality, 25, 70-87.

De Wied, M, Gispen-de Wied, C, \& van Boxtel, A. (2010). Empathy dysfunction in children and adolescents with disruptive behavior disorders. European Journal of Pharmacology, 626, 97-103.

Dissanayake, E. (2008). If music is the food of love, what about survival and reproductive success? Musicae Scientiae, 169-195. Special Issue.

Dolgin, KG, \& Adelson, EH. (1990). Age changes in the ability to interpret affect in sung and instrumentally presented melodies. Psychology of Music, 18, 87-98.

Eerola, T, \& Vuoskoski, JK. (2011). A comparison of the discrete and dimensional models of emotion in music. Psychology of Music, 39, 18-49.

Eisenberg, N. (1990). Prosocial Development in Early and mid-Adolescence. In R Montemayor, GR Adams, \& TP Gulotta (Eds.), From Childhood to Adolescence: A Transitional Period. Advances in Adolescent Development. An Annual Book Series, Volume 2. California: Sage.

Eisenberg, N, \& Fabes, RA. (1990). Empathy: Conceptualization, measurement, and relation to prosocial behavior. Motivation \& Emotion, 14, 131-149.

Eisenberg, N, \& Fabes, RA. (1999). Emotion, Emotion-Related Regulation, and Quality of Socioemotional Functioning. In L Balter \& C Tamis-LeMonda (Eds.), Child Psychology: A Handbook of Contemporary Issues (pp. 318-335). New York: Psychology Press.

Eisenberg, N, \& Miller, PA. (1987). The relation of empathy to prosocial and related behaviors. Psychological Bulletin, 101, 91-119.

Eisenberg, N, Lennon, R, \& Roth, K. (1983). Prosocial development: a longitudinal study. Developmental Psychology, $19,846-855$.

Eisenberg, N, Shell, R, Pasternack, J, Lennon, R, Beller, R, \& Mathy, M. (1987). Prosocial development in middle childhood: a longitudinal study. Developmental Psychology, 23, 712-718.

Eisenberg, N, Miller, PA, Shell, R, \& McNalley, S. (1991). Prosocial development in adolescence: a longitudinal study. Developmental Psychology, 27, 849-857.

Eisenberg, N, Carlo, G, Murphy, B, \& Van Court, P. (1995). Prosocial development in late adolescence: a longitudinal study. Child Development, 66, 1179-1197.

Feschbach, N, \& Feschbach, S. (2009). Empathyeducation. In J Decety \& W Ickes (Eds.), The Social Neuroscience of Empathy (pp. 85-97). Cambridge, Massachusetts: MIT Press.

Fredrickson, WE. (2000). Perception of tension in music: musicians versus nonmusicians. Journal of Music Therapy, $37,40-50$.

Fridja, NH. (1988). The laws of emotion. American Psychologist, 43(5), 349-358.

Gabrielsson, A, \& Lindström, E. (2001). The Influence of Musical Structure on Emotional Expression. In PN Juslin \& JA Sloboda (Eds.), Music and Emotion: Theory and Research (pp. 223-248). New York: Oxford University Press.

Gabrielsson, A, \& Lindström, E. (2010). The Role of Structure in the Musical Expression of Emotions. In PN Juslin \& JA Sloboda (Eds.), Handbook of Music and Emotion: Theory, Research, Applications (pp. 367-400). New York: Oxford University Press. 
Gallese, V. (2001). The shared manifold hypothesis. From mirror neurons to empathy. Journal of Consciousness Studies, 8 $(5-7), 33-50$

Garaigordobil, M. (2009). Comparative analysis of empathy in childhood and adolescence: Gender differences and associated socio-emotional variables. Journal of Psychology and Psychological Therapy, 9(2), 217-235.

Gohm, CL. (2003). Mood regulation and emotional intelligence: individual differences. Journal of Personality and Social Psychology, 84(3), 594-607.

Goodman, R, Meltzer, H, \& Bailey, V. (1998). The strengths and difficulties questionnaire: a pilot study on the validity of the self-report version. European Child and Adolescent Psychiatry, 7, 125-130.

Goodman, A, Lamping, DL, \& Ploubidis, GB. (2010). When to use broader internalising and externalising subscales instead of the hypothesised five subscales on the Strengths and Difficulties Questionnaire (SDQ): data from British parents, teachers and children. Journal of Abnormal Child Psychology, 38, 1179-1191.

Gross, JJ. (1998). The emerging field of emotion regulation: An integrative review. Review of General Psychology, 2(3), 271-299

lacoboni, M. (2009). Imitation, empathy, and mirror neurons. Annual Review of Psychology, 60, 657-670.

Isen, AM, \& Shalker, TE. (1982). The effect of feeling state on evaluation of positive, neutral, and negative stimuli: when you "accentuate the positive", do you "eliminate the negative"? Social Psychology Quarterly, 45(1),

John, OP, \& Srivastava, S. (1999). The Big-Five Trait Taxonomy: History, Measurement, and Theoretical Perspectives. In LA Pervin \& OP John (Eds.), Handbook of Personality: Theory and Research (Vol. 2, pp. 102-138). New York: Guilford Press.

Jolliffe, D, \& Farrington, DP. (2004). Empathy and offending: a systematic review and meta-analysis. Aggression and Violent Behavior, 9, 441-476.

Juslin, PN. (1997). Emotional communication in music performance: a functionalist perspective and some data. Music Perception, 14, 383-418.

Juslin, PN, \& Laukka, P. (2003). Communication of emotions in vocal expression and music performance: different channels, same code? Psychological Bulletin, 129, 770-814.

Juslin, PN, \& Laukka, P. (2004). Expression, perception, and induction of musical emotions: a review and a questionnaire study of everyday listening. Journal of New Music Research, 33(3), 217-238.

Juslin, PN, \& Timmers, R. (2010). Expression and Communication of Emotion in Music. In PN Juslin \& J Sloboda (Eds.), Handbook of Music and Emotion: Theory, Research, Applications. New York: Oxford University Press.

Juslin, PN, \& Västfjäll, D. (2008). Emotional responses to music: the need to consider underlying mechanisms. Behavioral and Brain Sciences, 31, 559-621.

Kastner, MP, \& Crowder, RG. (1990). Perception of the major/minor distinction: IV. Emotional connotations in young children. Music Perception, 8, 189-202.

Ladinig, O, \& Schellenberg, GE. (2012). Liking unfamiliar music: effects of felt emotion and individual differences. Psychology of Aesthetics, Creativity and the Arts, 6(2), 146-154.

Laiho, S. (2004). The psychological functions of music in adolescence. Nordic Journal of Music Therapy, 13(1), 49-65.

Liljeström, S. (2011). Emotional Reactions to Music: Prevalence and Contributing Factors. Unpublished Dissertation. Uppsala: Uppsala University. Department of Psychology.

Mayer, JD, \& Salovey, P. (1997). What is Emotional Intelligence? In P Salovey \& D Sluyter (Eds.), Emotional Development and Emotional Intelligence: Implications for Educators (pp. 3-31). New York: Basic Books.

Miller, PA, \& Eisenberg, N. (1988). The relation of empathy to aggressive and externalizing/antisocial behavior. Psychological Bulletin, 103, 324-344.

Miranda, D, Gaudrea, P, Debrosse, R, Morizot, J, \& Kirmayer, L. (2012). Music Listening and Mental Health: Variations on Internalizing Psychopathology. In R McDonald, G Kreutz, \& L Mitchell (Eds.), Music, Health and Wellbeing (pp. 513-530). New York: Oxford University Press.

Moore, BS. (1990). The origins and development of empathy. Motivation and Emotion, 14, 75-80.

Nawrot, ES. (2003). The perception of emotional expression in music: Evidence from infants, children, and adults. Psychology of Music, 31, 75-92.

North, AC, Hargreaves, DJ, \& O'Neill, SA. (2000). The importance of music to adolescents. British Journal of Education Psychology, 70, 255-272.

Punkanen, M, Eerola, T, \& Erkkilä, J. (2011). Biased emotional recognition in depression: perception of emotions in music by depressed patients. Journal of Affective Disorders, 130(1-2), 118-126.

Resnicow, JE, Salovey, P, \& Repp, BH. (2004). Is recognition of emotion in musical performance an aspect of emotional intelligence? Music Perception, 22, 145-158.

Ruud, E. (1997). Music and the quality of life. Nordic Journal of Music Therapy, 6(2), 86-97.

Saarikallio, S, \& Erkkilä, J. (2007). The role of music in Adolescents' mood regulation. Psychology of Music, 35(1), 88-109.

Saarni, C. (1999). The Development of Emotional Competence. NYC: The Guilford Press.

Salovey, P, Mayer, JD, Goldman, SL, Turvey, C, \& Palfai, TP. (1995). Emotional Attention, Clarity, and Repair: Exploring Emotional Intelligence Using the Trait Meta-Mood Scale. In JW Pennebaker (Ed.), Emotion, Disclosure, and Health (pp. 125-154). Washington, DC: American Psychological Association.

Salovey, P, Stroud, LR, Woolery, A, \& Epel, ES. (2002). Perceived emotional intelligence, stress reactivity, and symptom reports: Further explorations using the trait meta-mood scale. Psychology and Health, 17(5), 611-627.

Schubert, E, \& McPherson, GE. (2006). The Perception of Emotion in Music. In GE McPherson (Ed.), The Child as Musician: A Handbook of Musical Development (pp. 193-212). Oxford: Oxford University Press.

Swinkels, A, \& Giuliano, TA. (1995). 'The measurement and conceptualization of mood awareness: monitoring and labeling One's mood states. Personality and Social Psychology Bulletin, 21, 934-949.

Vieillard, S, Peretz, I, Gosselin, N, Khalfa, S, Gagnon, L, \& Bouchard, B. (2008). Happy, sad, scary, and peaceful musical excerpts for research on emotions. Cognition \& Emotion, 22, 720-752.

Vuoskoski, JK, \& Eerola, T. (2011a). The role of mood and personality in the perception of emotions represented by music. Cortex, 47, 1099-1106.

Vuoskoski, JK, \& Eerola, T. (2011b). Measuring music-induced emotion: a comparison of emotion models, personality biases, and intensity of experiences. Musicae Scientiae, 15, 159-173. 
Vuoskoski, JK, \& Eerola, T. (2012). Can sad music really make you sad? Indirect measures of affective states induced by music and autobiographical memories. Psychology of Aesthetics, Creativity, and the Arts, 6, 204-213.

Wöllner, C. (2012). Is empathy related to the perception of emotional expression in music? A multimodal time-series analysis. Psychology of Aesthetics, Creativity, and the Arts, 6(3), 214-223.

Zentner, M, \& Scherer, KR. (2008). Emotions evoked by the sound of music: characterization, classification, and measurement. Emotion, 8(4), 494-521.

doi:10.1186/s13612-014-0021-8

Cite this article as: Saarikallio et al:: Adolescents' expression and perception of emotion in music reflects their broader abilities of emotional communication. Psychology of Well-Being: Theory, Research and Practice 2014 4:21.

Submit your manuscript to a SpringerOpen ${ }^{\circ}$ journal and benefit from:

- Convenient online submission

- Rigorous peer review

- Immediate publication on acceptance

- Open access: articles freely available online

- High visibility within the field

- Retaining the copyright to your article

Submit your next manuscript at $\boldsymbol{\nabla}$ springeropen.com 